\title{
Field-responsive colloidal assemblies defined by magnetic anisotropy
}

\author{
Gabi Steinbach,,${ }^{1,2, *}$ Michael Schreiber, ${ }^{1}$ Dennis Nissen, ${ }^{3}$ Manfred Albrecht,${ }^{3}$ Ekaterina Novak,${ }^{4}$ Pedro A. Sánchez, ${ }^{4}$ \\ Sofia S. Kantorovich, ${ }^{4,5}$ Sibylle Gemming, ${ }^{1,2}$ and Artur Erbe ${ }^{2, \dagger}$ \\ ${ }^{1}$ Institute of Physics, Technische Universität Chemnitz, 09107 Chemnitz, Germany \\ ${ }^{2}$ Helmholtz-Zentrum Dresden-Rossendorf, Bautzner Landstrasse 400, 01328 Dresden, Germany \\ ${ }^{3}$ Institute of Physics, University of Augsburg, 86159 Augsburg, Germany \\ ${ }^{4}$ Ural Federal University, Lenin av. 51, Ekaterinburg, 620000, Russia \\ ${ }^{5}$ Computational Physics, Universität Wien, Sensengasse 8, Vienna, 1090, Austria
}

(Received 24 April 2019; published 18 July 2019)

\begin{abstract}
Particle dispersions provide a promising tool for the engineering of functional materials that exploit selfassembly of complex structures. Dispersion made from magnetic colloidal particles is a great choice; they are biocompatible and remotely controllable among many other advantages. However, their dominating dipolar interaction typically limits structural complexity to linear arrangements. This paper shows how a magnetostatic equilibrium state with noncollinear arrangement of the magnetic moments, as reported for ferromagnetic Janus particles, enables the controlled self-organization of diverse structures in two dimensions via constant and low-frequency external magnetic fields. Branched clusters of staggered chains, compact clusters, linear chains, and dispersed single particles can be formed and interconverted reversibly in a controlled way. The structural diversity is a consequence of both the inhomogeneity and the spatial extension of the magnetization distribution inside the particles. We draw this conclusion from calculations based on a model of spheres with multiple shifted dipoles. The results demonstrate that fundamentally new possibilities for responsive magnetic materials can arise from interactions between particles with a spatially extended, anisotropic magnetization distribution.
\end{abstract}

DOI: 10.1103/PhysRevE.100.012608

\section{INTRODUCTION}

Stimuli-responsive materials based on soft matter are applied widely in nowadays technologies. They exploit the structural arrangement of nanoparticles and microparticles in order to create specific (structural, electrical, ...) properties [1]. A large variety of techniques are available to fabricate particles with specific interactions [2-5]. For applications, the main challenge in particle synthesis is to trigger the interactions in a way such that particles self-assemble in desired structures. Functionality arises in such systems from the controlled formation of local configurations. Beyond that, functionality is extended if one can control the conversion between specific, well-defined structures in a reversible way [6]. This requires that a system can adapt versatile, well-defined local configurations under external manipulation in a controlled way [7].

The introduction of magnetic soft matter composites [soft materials with embedded magnetic particles (ferrofluids)] has greatly pushed forward the design and the performance of remotely controllable soft functional materials [8]. Magneticbased soft materials can be remotely aligned and guided under external fields $[9,10]$. In fact, magnetic fields of moderate strength combine several advantages over the use of, e.g., op-

\footnotetext{
*Present address: School of Physics, Georgia Institute of Technology, Atlanta, USA; gabi.steinbach.de@gmail.com

†a.erbe@hzdr.de
}

tical, electric, or acoustic fields to control structural assembly; they reach over large distances, achievable forces span a wide range, and their side effects on most natural materials are negligible [11]. As a consequence, prospective technical applications range from sensors to actuators and printed electronics [11-13]. Moreover, the compatibility of magnetic particles with living materials establishes unprecedented possibilities for clinical diagnostics, targeted drug delivery, and medical therapy [14-17].

Despite the many advantages, a major drawback of magnetic-based soft materials is the limited structural variability in magnetostatic equilibrium configurations, i.e., static structures with vanishing residual magnetic interaction forces between composing particles. The dominating dipole interaction between two magnetic particles leads to a collinear head-to-tail arrangement. Subsequently, in dipolar manyparticle systems, a combination of chains and rings form spontaneously (ferroparticles) $[18,19]$ or only chains form under homogeneous fields (ferromagnetic and paramagnetic particles) [10,20-23]. Beyond that, more diverse equilibrium patterns have been predicted and observed. Already for a few particles, one can find equilibrium, and even ground state, configurations of compact, onionlike structures where the dipoles form concentric rings [24]. Also, ferroparticles form networks through defects (branching points) under low temperatures [25-28]. In terms of applicability, the susceptibility to temperature of many materials may be limiting.

Structural variability in the self-assembly of ferrofluids can be crucially extended by introducing competing interactions 
(e.g., by thermodynamic, hydrodynamic, entropic, steric, or magnetic terms) that favor interparticle arrangements other than the dipole-induced head-to-tail configurations that lead to linear chains, rings, and compact structures. Inspired by the success in complex self-assembly of anisotropic particles [2, 29-31], nonlinear structures have been created with Janus particles, which exhibit a nonuniform magnetization distribution [32]. However, in often reported cases of Janus particles with laterally displaced dipoles, the underlying magnetic configuration remains the dipolar head-to-tail chain, and may lack reversibility if the formation requires in situ magnetization of the particles [22,33]. While such particles with high asymmetry (shift) can provide noncollinear order, they assemble in small clusters of up to six particles only [34,35].

The situation changes under conditions of selforganization, i.e., if the pattern formation is driven under a constant input of energy into the system. In such cases, the emerging patterns can vary depending on the energy input. This has been utilized with anisotropic paramagnetic particles under time-dependent fields. Competition between particle-field and particle-particle interactions enable the reversible formation of complex, tunable network structures [36]. However, the required material-specific magnetization dynamics inside the paramagnetic particles limits the choice of magnetic particles and defies generalization.

Better tunability via competing magnetic interactions is provided if ferromagnetic particles spontaneously selfassemble in a magnetically noncollinear manner already in the absence of external fields. This is already present in the compact, onionlike dipolar structures [24] or the defectinduced network structures [25,26] discussed above. Other promising candidates are ferromagnetic Janus particles that exhibit a specific, anisotropic magnetization [37-39]. If the magnetic center in these particles is shifted away sufficiently from the geometric particle center, the interparticle interaction favors the nonparallel arrangement, whereas the interaction of the particles with an external homogeneous field favors the parallel one [40]. Tuning the ratio between both interactions enables control over structural complexity and assembly rates, which fundamentally extends design flexibility.

Starting from colloidal networks that form as a conse-quence of a bistable self-assembly behavior [37], we demonstrate that we can generate a variety of field-tuned, colloidal structures in a controlled and reversible way. We employ magnetic Janus particles with a ferromagnetic coating on one hemisphere $[37,40]$ while the other hemisphere is nonmagnetic [Fig. 1(a)]. In contrast to other, often studied magnetic Janus particles with lateral dipole shift [22,34,35], our particles are characterized by an extended magnetization distribution with the magnetic anisotropy pointing perpendicular to the particle surface. This enables the untypical network formation. We study the influence of both static in-plane $B^{\text {ip }}$ and out-of-plane $B^{\text {op }}$ external fields on the colloidal self-assembly. The experimental findings are complemented by molecular dynamics (MD) simulations, performed on a system of colloidal particles with a symmetric arrangement of radially shifted dipoles.

The results demonstrate that the diversity and reversibility of structure formation under external fields can be increased by two ingredients, magnetic inhomogeneity (shift) and the (a)

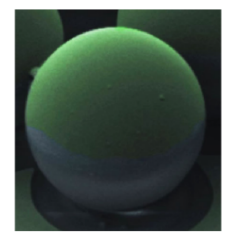

(b)

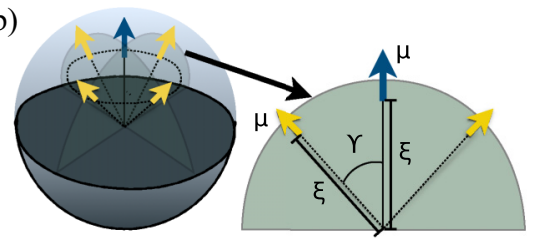

FIG. 1. Illustrations of the experimental Janus particle and the $5 s d$ particle model. (a) Scanning electron microscopy image of a colloidal silica particle with the hemispherical magnetic thin film illustrated green. (b) Simulation model of a $5 s d$ particle, a sphere with five shifted dipoles, a central one (blue), and four side dipoles (yellow), with enclosed angle $\gamma$ and radial shift $\xi$.

spatial extension of the magnetization distribution inside the ferroparticles.

\section{MATERIALS AND METHODS}

\section{Experimental details}

We study silica particles (radius of $2.27 \mu \mathrm{m}$ ) with a magnetic thin film coating on one hemisphere [Fig. 1(a)]. Film deposition follows the routine described previously [41]. The magnetic thin film consists of a multilayer stack of $\mathrm{Ta}(3.0 \mathrm{~nm}) / \mathrm{Pd}(3.0 \mathrm{~nm}) /[\mathrm{Co}(0.28 \mathrm{~nm}) / \mathrm{Pd}(0.9 \mathrm{~nm})]_{8} / \mathrm{Pd}$ $(1.1 \mathrm{~nm})$. The bottom Ta layer enhances bonding between the metallic layer and the silica sphere. The top 2.1-nm layer of Pd prevents oxidization of the metallic film. The alternating Co and Pd layers provide the magnetic properties of the stack. They exhibit perpendicular magnetic anisotropy, i.e., the magnetic flux points out of the film plane. When deposited on the spherical particles, after magnetic saturation such a film provides a rotationally symmetric magnetization distribution, and the far field exhibits dipolar characteristics. The resulting permanent magnetic moment of about $m=$ $6.4 \times 10^{9} \mu_{\mathrm{B}}$ points perpendicular to the Janus interface. As a consequence, the ratio between dipole interaction between particles in contact and thermal energy can be estimated as 900 , inducing forces that are strong enough to guarantee longtime stability of self-assembled clusters at room temperature. Our particles contrast with magnetic Janus particles with in-plane magnetization, where the magnetic moment points parallel to the Janus interface [22,35]. In addition, the particles in our study exhibit fixed magnetic properties during the experiments; the applied magnetic fields and the magnetic particle-particle interaction are too weak to alter the magnetic state of the individual beads (see Appendix, Fig. 8).

We use videomicroscopy to study the self-assembly. Particles are dispersed in water. The silica particles with a density of $2 \mathrm{~g} / \mathrm{cm}^{3}$ rapidly sediment on the planar glass substrate due to the density mismatch with water $\left(1 \mathrm{~g} / \mathrm{cm}^{3}\right)$, providing a two-dimensional system. For studies of field-tuned particle assembly, we apply a homogeneous static field $B^{\text {ip }}$ in the substrate plane and an oscillating field with amplitude $B^{\text {op }}$ at a frequency of $5 \mathrm{~Hz}$ perpendicular to it. The amplitude of both fields, created by a set of three mutually orthogonal coils, is up to $3 \mathrm{mT}$. In all our experiments, we compensated for the earth magnetic field. 


\section{B. Numerical details}

Equilibrium MD simulations are carried out with the simulation package ESPRESSO[42,43]. We use the Langevin thermostat. The value of the reduced temperature, which is equal to the ratio of the actual thermal energy to the energy scale of the short-range interaction in our system $\left(\epsilon_{s}=1\right)$, is set to unity $T=1$. Using the inertial Langevin dynamics, we can avoid the explicit simulation of the solvent, and restrict the effects of the thermal fluctuations of the carrier fluid on the colloidal particles to stochastic terms added to the translational and rotational equations of motion [44]. Hydrodynamic effects are ignored in our simulations. As a result, we put the friction coefficient to unity, as well as particle masses.

Each Janus particle in our simulations is composed of a soft sphere with radius $\sigma$, which provides the measure for the length scale in the system. Thus, neighboring particles interact with a soft core steric repulsion, given by a Weeks-ChandlerAndersen potential [45]. To mimic the magnetic cap, the model sphere is equipped with an off-center, radially symmetric arrangement of four shifted dipoles around a central, equally shifted one [Fig. 1(b)]. The dipoles are implemented as virtual sites [43], and point radially outward. The dipoles with moment $\mu$ are shifted by a value $\xi$, measured as fraction of particle radius $\sigma$. The central dipole and the side dipoles enclose an angle $\gamma$. Such a "5 shifted-dipole particle" (5sd particle) is a natural minimal extension of previous work on particles with only two shifted side dipoles ( $3 s d$ particles) $[35,46,47]$. The extension to four side dipoles better models the rotational symmetry of the experimental particles, which becomes relevant for the out-of-plane rotations. During the simulations, we impose a quasi-two-dimensional (quasi-2D) geometry by keeping the centers of all particles in one plane. Accordingly, all translations are limited to that plane, whereas the rotations are allowed in full three-dimensional (3D) space.

External fields are applied in the simulation in two different ways. In the case of the in-plane field, we either increase it slowly in a stepwise manner, or we quench the system by switching on the field to its maximum value. The out-of-plane field is always switched on to its maximum value. The area fraction covered by $N=512$ particles is $\phi_{a}=N s_{p} / S=0.08$, with the particle cross section $s_{p}$ and the area of the layer $S$. We always apply lateral periodic boundary conditions. In order to efficiently calculate the magnetic interactions under periodic boundaries in slab geometry, we chose the dipolarp3m algorithm and dipolar layer corrections [48,49].

\section{RESULTS AND DISCUSSION}

With the numerical and experimental ingredients of magnetic Janus particles with extended magnetization distribution, we run several sets of investigations. First, we study selfassembly in the absence of external fields to derive the optimal values for the model parameters that describe the experimental particles; next, we study exclusively the influence of an inplane field $B^{\text {ip }}$ (parallel to the self-assembly plane) on the structure formation; and, finally, the impact of an out-of-plane field $B^{\text {op }}$ (perpendicular to the self-assembly plane) is investigated. Under these different conditions we have observed the reversible formation of the various structures shown in Fig. 2, (a) type I
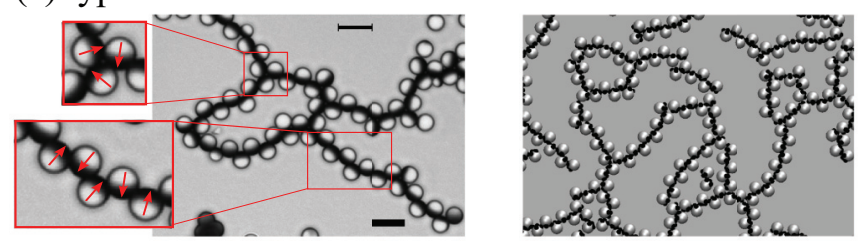

(b) type II

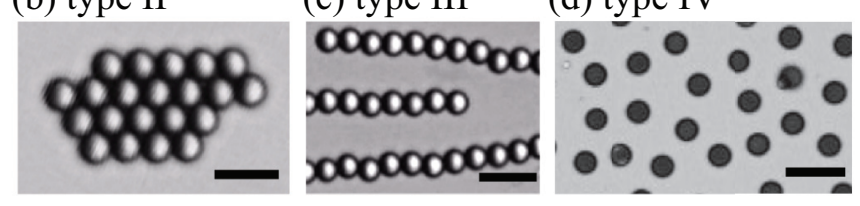

FIG. 2. Self-assembled structures of the capped colloidal particles. (a) Microscopy image (left) and simulation snapshot (right) of self-assembled Janus particles. Open structures are built from staggered chains and branching points (insets) ( $5 s d$-model parameters: $\mu=0.9 ; \gamma=6 \pi / 25 ; \xi=0.94)$. (b)-(d) Microscopy images of field-induced self-organization of (b) compact clusters, (c) linear chains, and (d) single particles. Scale bar: $10 \mu \mathrm{m}$.

which will be discussed and labeled as type I to type IV in the following.

\section{A. Field-free self-assembly}

The capped Janus particles form clusters spontaneously [Fig. 2(a)] as a consequence of the long-range magnetic interaction between them. Untypical for magnetic particles, the clusters are networks. They consist of triangular configurations that serve as branching points to which staggered chains are attached [see insets in Fig. 2(a), left]. Previously, we discovered that this formation is a consequence of a bistable self-assembly behavior; three capped particles can self-assemble in two structurally distinct configurations [37]. One is a staggered chain with antiparallel cap orientation of neighboring particles, which exhibits a total magnetization that points perpendicular to the chain backbone. The other one is a triangular configuration, in which the magnetic caps form a flux-closure ring and, thus, have zero total magnetic moment. This bistability during self-assembly manifests itself in the formation of networks as depicted in Fig. 2(a).

Bistability in a single-component system, providing the formation of networks based on distinct structural elements with different magnetic properties, is unusual. Dipolar systems, for example, can also form percolating networks, but the dipoles adopt configurations which are close to head-to-tail orientation, both in linear segments and branching points [50]. Networks formed via distinct structural motifs have so far been observed for multicomponent systems only [51,52]. In addition, it should be noted that the staggered chains optically resemble those shown in previous reports based on capped Janus particles with in-plane magnetization [22,53]. However, they crucially differ in their magnetic configuration: While those chains preserve the dipolar head-to-tail arrangement, in our chains the dipoles are not collinear. This crucial difference becomes relevant later for field-tuned transformations.

Previous numerical analysis showed that the bistability is a consequence of a broad, anisotropic magnetization 
(a)
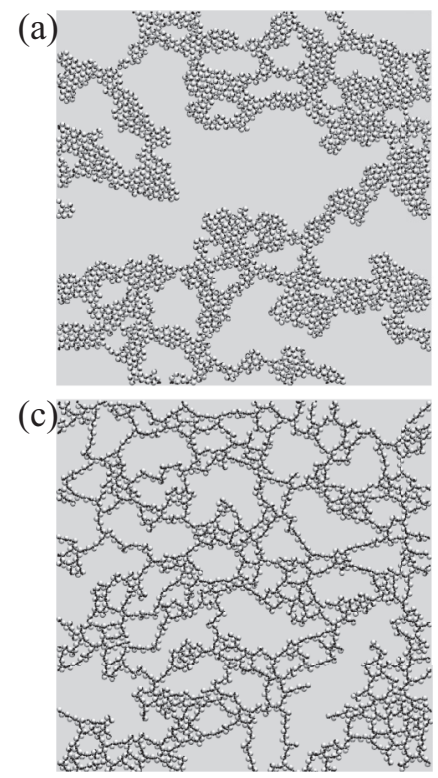

(b)

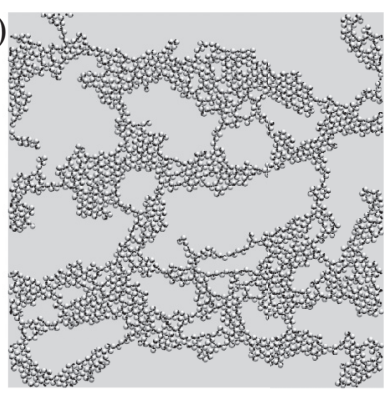

(d)

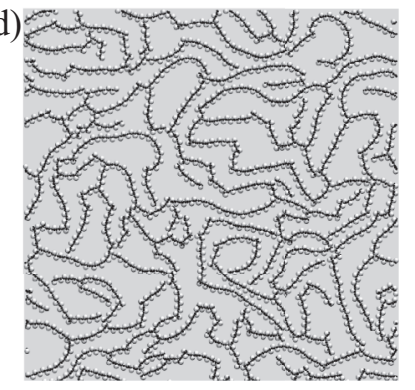

FIG. 3. Simulation snapshots of self-assembled structures of $5 s d$ particles for various dipole shifts $\xi$ : (a) 0.82 , (b) 0.86 , (c) 0.90 , (d) $0.94(\mu=0.9 ; \gamma=6 \pi / 25)$

distribution of the Janus particles discussed here [37]. Numerical simulations of 2D assemblies with $3 s d$ particles have revealed that bistability occurs only for magnetization distributions represented by an intermediate range of magnetic shifts and distribution widths [Fig. 2(a), right], which are described by the parameters $\xi, \gamma$ shown in Fig. 1(b). For small shift values $\xi$ and narrow distributions, given by small values of $\gamma$, the triangular configuration becomes dominant, whereas for high shifts and wide distributions only staggered chains form. Here, we employ the $5 s d$ particle model to analyze the network formation. We set $\gamma=6 \pi / 25$, which ensures bistability for a broad range of shifts $\xi$ according to our previous analysis of small clusters [37]. For simplicity, we set equal shifts $\xi$ and dipole moments $\mu$ for central and side dipoles. The value of $\mu$ was set to 0.9 in numerical dimensionless units. On the one hand, this choice provides a sufficiently large dipolar interaction between the particles as compared to thermal noise to represent the experimentally observed cluster stability. In fact, one can estimate the effective moment of a $5 s d$ particle to be approximately equal to 3.5 and an effective shift to be approximately 0.7 , which provides us with the highest absolute value of the interaction energy on the order of 60. This upper limit though is never reached as the discrete distribution of dipoles has very strong short-range effects. On the other hand, it sets the scale for the dimensionless applied magnetic field discussed below. Moreover, in the previous studies [37], we showed that only the ratio between the dipole values matters. As we are not aiming at a quantitative comparison with the experiment, the energy scale in the simulation was chosen to provide a fast convergence to the equilibrium state. Finally, the dipole shift $\xi$ remains as the only adjustable parameter to control structure formation.

Networks formed by $5 s d$ particles with various values of $\xi$ are presented in Fig. 3. For a stepwise increase of $\xi$, a striking evolution from compact clusters to "empty" networks can be

observed. Simulated and experimentally observed structures agree best for $5 s d$ particles with a shift value of $\xi=0.94$. This choice emerges when comparing the distribution of coordination numbers $N$ (the number of nearest neighbors a particle has) between experimental clusters and numerical clusters with various values of $\xi$ (see Appendix, Fig. 9). From this analysis we conclude that particles with a shift value of $\xi=0.94$ best represent the experimental spread of coordination numbers.

Since the field-free self-assembly of the Janus particles provides a noncollinear orientation of their dipole moments, homogeneous external fields induce a change of the magnetic ordering when the particles align along the field. This in turn gives rise to translational rearrangements within the clusters due to the anisotropy of the magnetic interparticle interaction. Applying constant parallel and perpendicular fields, we experimentally observed transformations between different structures, which will be presented in the following.

\section{B. Self-organization under in-plane fields}

Fields $B^{\text {ip }}$ parallel to the assembly plane induce a continuous transformation from open networks [type I, Fig. 4(a), left] to close-packed structures [type II, Fig. 4(a), right] [54]. Under small external fields, the particle-particle interaction is predominant and the aforementioned network structure is retained. Particles in the networks are characterized by a coordination number $N<4$. Upon increasing $B^{\text {ip }}$, the caps gradually rotate in the substrate plane to align with the field. While particles in branching points do not rearrange under this alignment, staggered chains respond with a continuous contraction.

The transition can be clearly seen in a short, nonbranched staggered chain of the capped Janus particles, as presented in snapshots in Fig. 4(b) and in a movie of the chain under applied fields [54]. With increasing $B^{\text {ip }}$, the chain continuously contracts. The stagger angle [red vertex in Fig. 4(b)] depends on the intensity of the external in-plane field and can reversibly be set between about $120^{\circ}$ in the field-free case and $60^{\circ}$ for $B^{\text {ip }}$ (fully contracted) at about $0.12 \mathrm{mT}$. In compact chains, particles obtain an upper limit of local coordination numbers as $N=4$. Broader close-packed clusters of type II [Fig. 4(a), right] with $N>4$ form only from initially branched structures. They form through lateral combination of the chains at a branching point during chain contraction [54]. Above $B^{\text {ip }}=0.18 \mathrm{mT}$, only double-stranded chains and broader compact clusters (type II) exist.

It should be noted that the field-induced formation of compact clusters is very stable against slight mechanical disturbance and, thus, differs fundamentally from compaction by van der Waals interactions, which has been reported earlier [55]. We can, thus, exclude van der Waals interactions as the driving forces for the formation of compact clusters in our system. Similar compact clusters have also been observed for paramagnetic particles in parallel fields [56]. In fact, the compact formation of collinear, dipolar particles is energetically favorable over the state of separate, linear chains $[19,56]$. Experimentally, such compact clusters form through the lateral aggregation of linear chains. It requires mechanical or thermal excitation to overcome the energy barrier of dipolar repulsion 
(a)

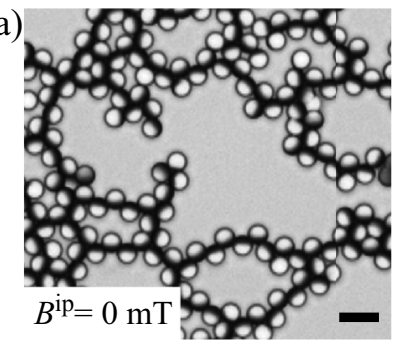

(b)

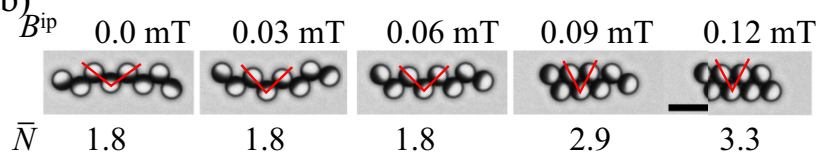

(c)

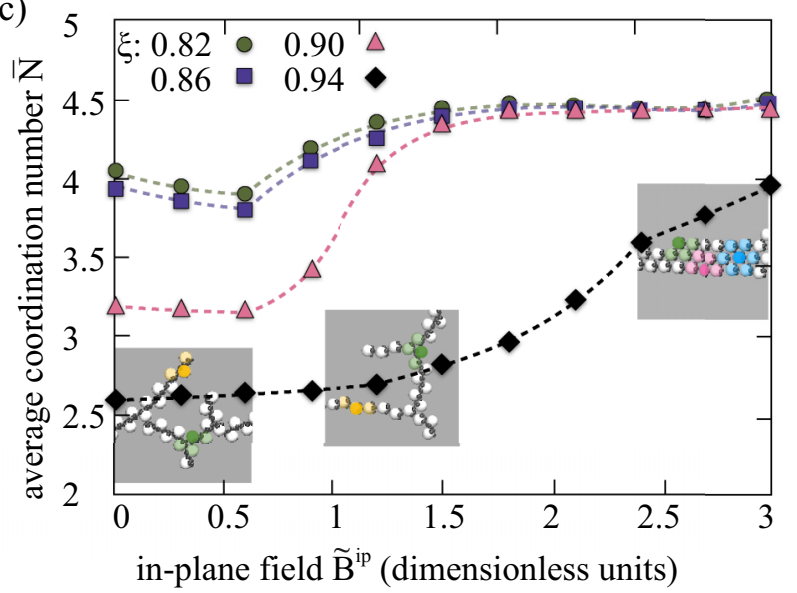

FIG. 4. Clusters align and pack under in-plane fields $B^{\text {ip }}$. (a) Microscopy images of a cluster before (left) and after (right) a saturating in-plane field has been applied. Scale bar: $10 \mu \mathrm{m}$. (b) Reversible transformation of staggered chains into double chains under $B^{\text {ip }}$. (c) Average coordination number of a particle as a function of $B^{\text {ip }}$ for various values of $\xi$. Insets show simulation snapshots of typical clusters for $\xi=0.94$ at corresponding field values. Colors indicate particles that have two (yellow), three (green), four (pink), or six neighbors (blue).

at larger chain distances, which is reported to take hours. In contrast, in our system the field-free equilibrium state of a network structure enables the instant formation of compact clusters within seconds after the in-plane field is switched on.

As a further feature of the particle system, the presented process of cluster compaction I $\rightarrow$ II is fully reversible. After releasing $B^{\text {ip }}$, branched clusters form again (II $\rightarrow$ I). The reversibility of the field-controlled reconfiguration is striking for the staggered chain as depicted in Fig. 4(b) and shown in Ref. [54]. After contraction, double-stranded chains reversibly expand into staggered chains via the reorientation of the caps from parallel to antiparallel. Such a process provides a microscopic contractile module that can be remotely controlled. The presented continuous control over the the stagger angle and cluster compaction is a unique feature of our system that results from the noncollinear networks that form under equilibrium conditions.

In order to show that such structures and their reversible interconversion result purely from competing magnetic interactions, we employ MD computer simulations of the in-plane-field-induced self-assembly, where apart from fielddipole and dipole-dipole interactions only short-range repulsion between colloids is taken into account.

Figure 4(c) displays the evolution of the average coordination number per particle $\bar{N}$ as a function of slowly growing strength of the dimensionless, numerical in-plane field $\widetilde{B}^{\text {ip }}$. For a dipole shift of $\xi=0.94$, the value of $\bar{N}$ steadily grows with field strength. This suggests that clusters contract upon field application, which agrees with the experimental findings. It is also visualized by snapshots of typical clusters at three different field values in the insets of Fig. 4(c). Apart from the shift value $\xi=0.94$, which best describes the experimental particles, we also plot $\bar{N}$ for systems with smaller values of dipolar shift: $\xi=0.82,0.86$, and 0.9 [see Figs. 3(a)-3(c)]. For smaller shifts, the particle coordination number is always larger and changes only slightly with the field strength. This is natural as $5 s d$ particles with small values of $\xi$ already favor compact structures in the absence of fields [see Fig. 3(a)], which changes their response to external fields. In the case of $\xi=0.82$, the in-plane field causes the particles to only rotate in the already formed compact cluster, only slightly increasing the average number of nearest neighbors. In contrast, for $\xi=0.94$, initially staggered chains, in which particles have two neighbors, contract under the applied field.

The process of cluster contraction I $\rightarrow$ II is also reversible in the numerical system. However, while the cluster expansion from compact into staggered chains is obtained in the numerical system, it differs qualitatively from the experimental observations. Once the field $B^{\text {ip }}$ is switched off, parallel arrangements of head-to-tail oriented chains dissolve, and the process of staggered chain formation starts over. Note, however, that the two end configurations of the transformation do not depend on such details of the transformation.

We can conclude that in our single component system compact and open, branched clusters can be created and converted into each other by in-plane fields in a well-defined way. Such a possibility to precisely control and reverse the topology of the clusters is not present, for example, in staggered chains of magnetic Janus particles where the resulting magnetic moment points parallel to the Janus interface. There, the magnetic hemispheres form dipolar head-to-tail chains, and the staggered configuration of the dipolar chains follows from the excluded volume of the nonmagnetic hemispheres. In such systems, different stagger angles are realized nonreversibly by either using particles with different magnetic content [21] or by in situ magnetization of the caps [22]. In our system, the nonparallel orientation of neighboring particles in the staggered chain competes with the particle-field interaction, which uniformly aligns the particles. The competition allows for a tunable stagger angle and enables reversible. On top of that, broader compact clusters are achievable through the second structural pattern, the rotational rings that serve as branching points, where contracting staggered chains combine laterally.

Finally, one needs to underline that the structure of the clusters observed in in-plane fields of an intermediate strength is very similar to that observed in particle systems with centrally positioned magnetic moment [57]. The reason for that is the Zeeman energy which becomes dominant over the 
(a) I↔III $\leftrightarrow$ IV

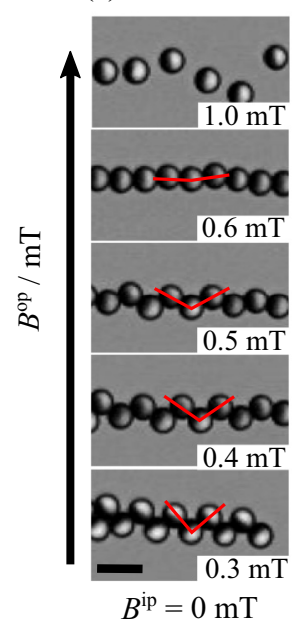

(b) II $\rightarrow$ III $\leftrightarrow$ IV

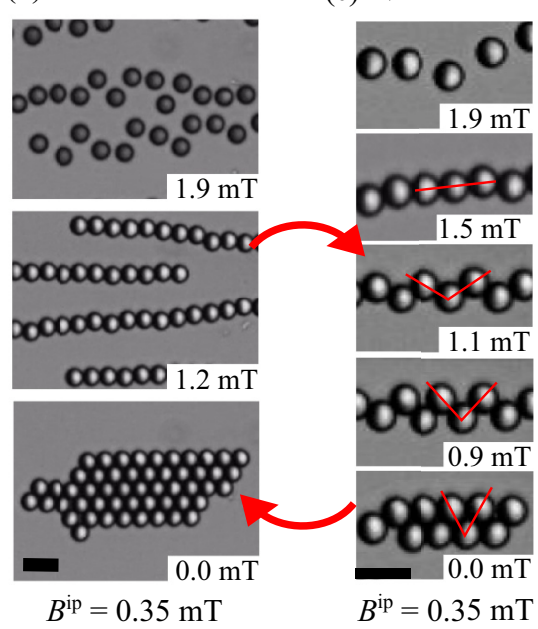

FIG. 5. Microscopy scenes of field-induced transformations. (a) Reversible expansion of a staggered chain under increasing field amplitudes $B^{\text {op }}(\omega / 2 \pi=10 \mathrm{~Hz})$. (b) Transformation of a compact cluster into lines and single particles under increasing amplitudes $B^{\text {op }}$ $(\omega / 2 \pi=10 \mathrm{~Hz})$, while a constant field $B^{\text {ip }}$ is applied [54] and (c) its reversion via formation of a single chain followed by contraction into a double chain under reduction of $B^{\text {op }}$. The transition II $\rightarrow$ III $\rightarrow$ II in (b) and (c) can be achieved only following the direction indicated by the red arrows. Scale bar: $10 \mu \mathrm{m}$.

magnetic interparticle interactions and suppresses the effects caused by the internal particle magnetic anisotropy.

\section{Self-organization under out-of-plane fields}

The application of fields $B^{\text {op }}$ perpendicular to the assembly plane provides an additional possibility to control structure formation. It imposes an out-of-plane component on the particle orientations. Here, we apply oscillating perpendicular fields to prevent particles from piling up in the vertical direction, retaining a 2D assembly plane with 3D particle orientation. At low field frequency, such oscillations have no qualitative consequences for the observable 2D structures, and we may focus on the structural transformation induced by different amplitudes of $B^{\text {op }}$.

The experimental diagram in Fig. 5 shows structural transformations that can be realized under perpendicular fields $B^{\mathrm{op}}$. Staggered chains and networks (I) continuously transform into linear, single-stranded chains (type III) [Fig. 5(a)] [54]. We have shown previously that this reconfiguration, which is initiated by a continuous alignment of the caps from antiparallel to parallel, results from the magnetic interaction of particles with off-center dipoles under an oscillating field [40]. Upon reducing $B^{\text {op }}$, the transition is reversible. Branching points and staggered chains form again. For even higher fields $B^{\text {op }}$, linear chains abruptly break apart into single particles (type IV) as shown in Fig. 5(a). This transformation is fully reversible when decreasing $B^{\text {op }}$.

The self-assembly scenario changes when first a saturating constant in-plane field $B^{\text {ip }}$ is applied and then the field $B^{\text {op }}$ is added. We observed that the compact clusters (type II) obtained in a saturating field $B^{\text {ip }}$ separate into single-stranded

chains when an additional field $B^{\text {op }}$ is applied [transition II $\rightarrow$ III, Fig. 5(b)]. During the transition from compact clusters to linear chains, all caps remain parallel. Thus, compact structures also undergo a transition to head-to-tail chains if they are exposed to an out-of-plane field. This transition is reversible, albeit takes place via different paths for decreasing (III $\rightarrow$ II) and increasing (II $\rightarrow$ III) out-of-plane fields. If $B^{\text {op }}$ increases, compact clusters simply break into parallel chains. Following that, if the out-of-plane field decreases, individual chains first rearrange themselves to form double-stranded chains with parallel orientation of the caps (a compact cluster with only two lines) and then start collapsing into larger compact configurations [Fig. 5(c)] [54].

Previously reported long-time experiments have shown that linear chains of dipolar particles can aggregate laterally [56] when the thermodynamic equilibrium is reached. This requires that the chains overcome a magnetostatic energy barrier. In our presented system, the thermal energy is orders of magnitude smaller than the magnetic interaction. Hence, we do not expect such a lateral aggregation for experimentally realizable long times.

Finally, once linear chains (type III) have formed under $B^{\text {op }}$, one can further increase the field intensity. This leads to a separation of the linear chains into single particles (type IV). The separation can be reversed by reducing $B^{\text {op }}$ (or increasing $B^{\text {ip }}$ accordingly).

In numerical studies, we examine whether the experimentally observed transitions in combined in-plane and out-ofplane fields can result from the interplay between particlefield and particle-particle magnetic interactions only. We investigated structural transitions taking place in the system of $5 s d$ particles under an out-of-plane field with different amplitudes while a constant, saturating in-plane field $\left(\widetilde{B}^{\text {ip }}=3\right.$, in simulation dimensionless units) was applied. Here, we will focus exclusively on systems with $\xi=0.94$.

We extract the fractions of single particles (coordination number $N=0)$, particles aggregated in chains $(N=1,2)$, and those in compact configurations $(N>2)$ as a function of the amplitude of the out-of-plane field [Fig. 6(a)]. With increasing field amplitudes, all three fractions change continuously. Compact structures gradually vanish, getting replaced by chain structures. The latter obtain a maximum at intermediate fields, followed by a decay. The fraction of single particles continuously increases and eventually becomes dominant. We can conclude that at zero out-of-plane field, almost all particles are grouped in large compact clusters [right inset in Fig. 4(c)]. Increasing $\widetilde{B}^{\text {op }}$ causes compact structures to transform first into chains and then separate into single particles. We can see that starting from $\widetilde{B}^{\text {op }} \sim 2.7$ [intersection between orange and blue curves in Fig. 6(a)], more particles are found in chains than in compact clusters. At about $\widetilde{B}^{\text {op }}>3.6$, chains start to disappear and in the further course the majority of particles become single.

The analysis of the coordination number at different outof-plane amplitudes provides insight into the change in topology of clusters. To verify that these changes are associated with cluster breakup, we characterize the structural transitions observed in this specific set of simulations in more detail by plotting cluster size distributions at three different values of the out-of-plane amplitude [Fig. 6(b)]. We choose a binary 

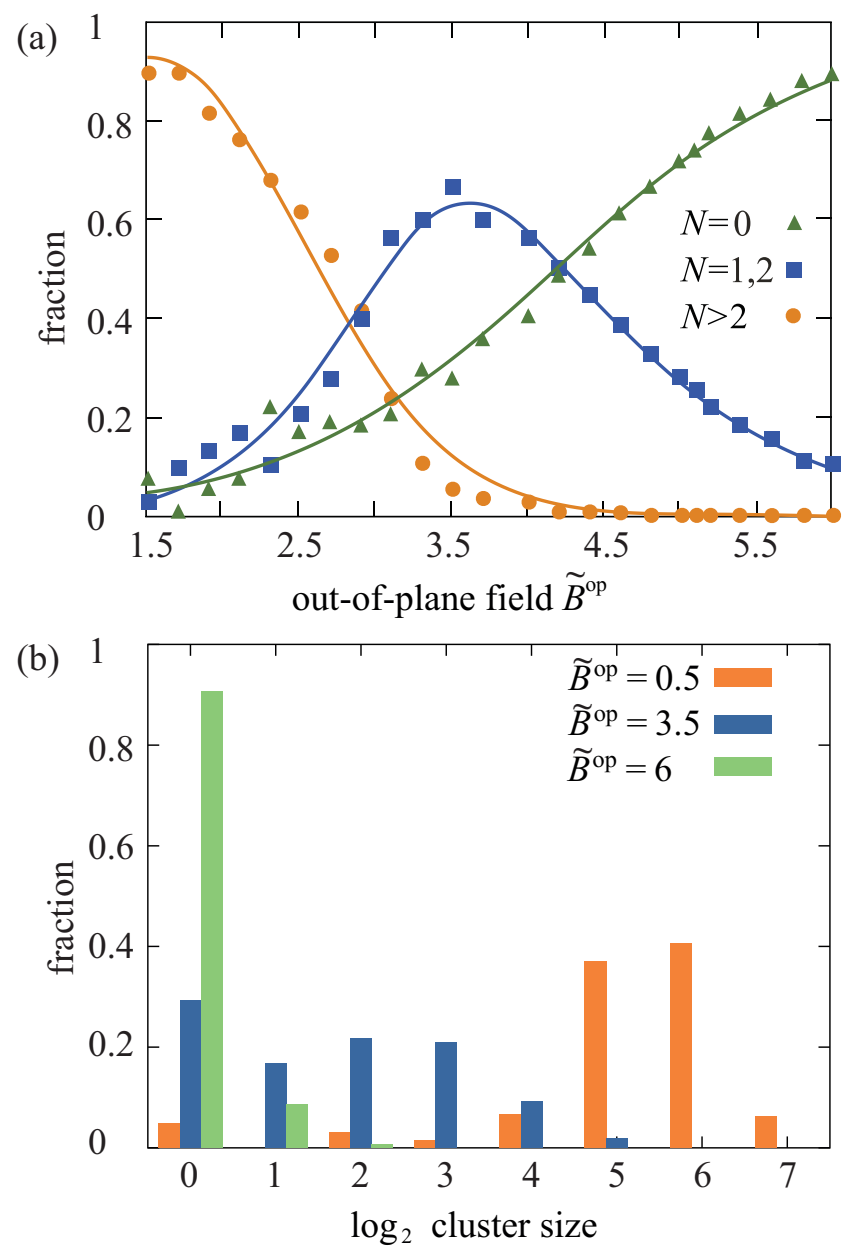

FIG. 6. Structural characterization of simulated clusters of $5 s d$ particles under a saturating in-plane field $\left(\widetilde{B}^{\text {ip }}=3\right)$ at various out-ofplane fields $\widetilde{B}^{\text {op }}$. (a) Fractions of particles with different coordination numbers $N$ (see legend) as a function of $\widetilde{B}^{\text {op }}$. Lines are least-squares fits, shown to guide the eyes. (b) Cluster size histogram for three different values of $\widetilde{B}^{\text {op }}$ (see legend). Note the logarithmic scale of the cluster size axis.

logarithmic scale for the cluster size and group clusters with $2^{x}-1<$ cluster size $<=2^{x}$ at $x \in \mathbb{Z}$. At a small field value of $\widetilde{B}^{\text {op }}=0.5$, in a saturating in-plane field, clusters are very large, ranging up to 150 particles per cluster. For the intermediate field amplitude where chain structures are dominant, one sees a noticeable decrease in the total cluster size as well as in the size distribution. Finally, at $\widetilde{B}^{\text {op }}=6$, a clear majority of particles are single. Overall, our simulations qualitatively reproduce the experimentally observed structures presented [Figs. 5(b) and 5(c)] for the case of saturating in-plane fields and at various out-of-plane fields. This clearly supports the statement that the magnetic interactions play the dominant part in the experimentally observed structural transitions.

\section{CONCLUSIONS}

We have presented experimental results on the steerable, rich self-assembly behavior of magnetically anisotropic particles in two dimensions under external fields. Starting from

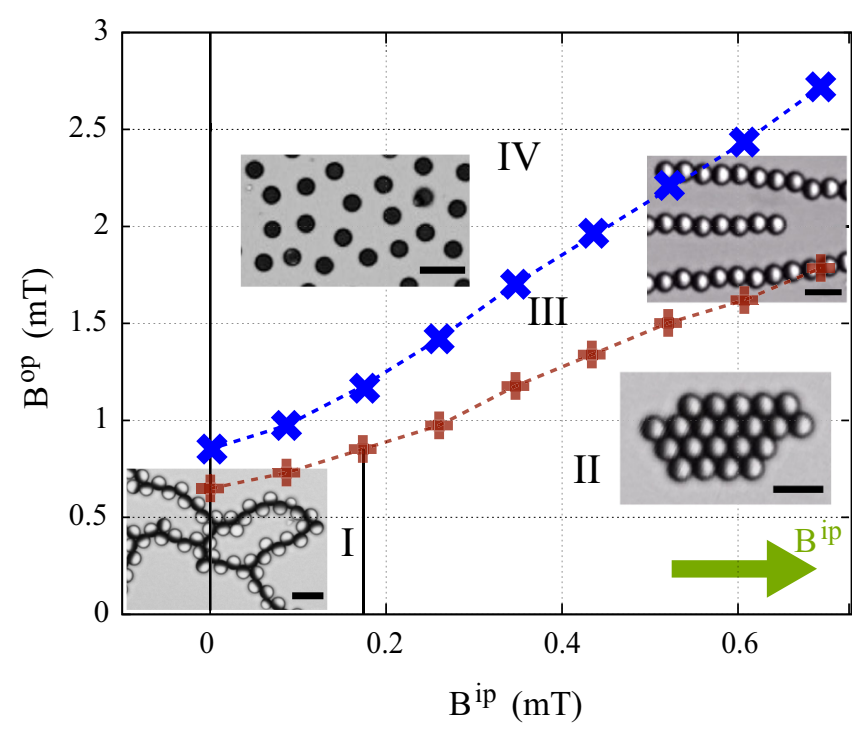

FIG. 7. State diagram of magnetic colloidal clusters. Static inplane fields $B^{\text {ip }}$ (horizontal direction) generate a transformation between branched (type I) and compact (type II) clusters and out-ofplane fields $B^{\text {op }}$ enable transformations from branched and compact clusters (types I, II) to linear chains (type III) and single particles (type IV). Dotted curves are guides to the eye. Scale bar: $10 \mu \mathrm{m}$.

a network structure consisting of bistable motifs with noncollinear magnetic arrangement, constant parallel and perpendicular fields are used to create and interconvert a diversity of structures by magnetic means only. The observed structures, which have been labeled from I to IV, can be summarized and displayed in a state diagram (Fig. 7). The structures can be converted into each other via magnetic fields, where they undergo structural reorganizations as presented in Figs. 4 and 5. The presented transformations are reversible at any point in the state diagram. In analogy to the simulations [Fig. 6(a)], the field-induced transitions in the experimental system take place gradually with increasing field. The range of applied field from the onset of the transition until its completion is quite small compared to the numerical results, being about $0.1 \mathrm{mT}$ only. The data points in Fig. 7 mark the points where the transformation is completed.

With these findings, we demonstrate that magnetic selforganization can be fundamentally extended if the particles spontaneously form magnetically noncollinear assemblies, which contrasts with typical dipolar particles. Noncollinear order can be realized if the particles exhibit a radially shifted magnetic center and an extended magnetization distribution. These magnetic properties lead to the formation of branched, staggered chains with nonparallel magnetic moments by selfassembly of a single particle species. The alignment of the particles under external fields provides controlled, reversible transformations between diverse structures. At low field intensities, a combination of $B^{\text {ip }}$ and $B^{\text {op }}$ allows the reversible adjustment of the bond angle in staggered chains over the whole range from compact, double-stranded chains (Fig. 4) to singlestranded, linear chains. Moreover, by MD simulations of simplified model spheres with five radially shifted point dipoles, we have shown that the stability and the transformations of 
the experimentally observed structures are solely based on magnetostatic interactions between the particles. The fact that all presented transitions are found to be reversible also in simulations evidences that while hydrodynamic interactions and friction stretch the experimental relaxation timescales, they do not qualitatively affect the topology of the resulting clusters.

The simplicity of the numerical model of $5 s d$ particles suggests that the findings can be transferred to other systems of widely discussed anisotropic magnetic particles [58]. In addition, initially nonmagnetic objects, such as cells, could be employed after embedding magnetic components in an anisotropic manner using recently promoted synthesis techniques based on microfluidics [59-61] or surface modification $[41,62,63]$.

The presented precise control of structure formation and reconfiguration under low external fields provides potential use in responsive materials for highly sensitive magnetic and optical sensors or micromechanical actuators. The magnetization of the material arises from the distribution of the particle orientations, which is directly linked to the particle positions. The orientational configuration could additionally be used for tunable transmission or even for optical polarization if substrate spheres are made of optically active materials [64,65]. For micromechanical applications, the reversible expansion and contraction of the assemblies, particularly of the staggered chains, provides a novel control strategy with precise tuning.

\section{ACKNOWLEDGMENTS}

Financial support from the German Research Foundation (DFG, Grants No. ER 341/9-1, No. FOR 1713 GE 1202/91 , and No. AL 618/11-1) is gratefully acknowledged. This research has been supported by the Russian Science Foundation Grant No. 17-72-10145. S.S.K. acknowledges support from the Austrian Science Fund (FWF), START-Projekt No. Y 627-N27. G.S. acknowledges funding from the Academy of Sciences Leopoldina (Grant No. LPDS 2017-03).

\section{APPENDIX}

\section{Magnetization of capped spheres in external fields}

The presented work on controlled self-organization employs the alignment of magnetically capped Janus particles in external fields. The explanation for the reconfiguration of the particles is based on the assumption that the particles exhibit a fixed anisotropy distribution with a net magnetic moment. This assumption presupposes that the stray field of neighboring particles as well as the externally applied fields have no influence on the anisotropy distribution. To verify this assumption, we have performed magnetometry measurements of the particles under external fields.

For magnetometry measurements, we prepare the sample as described in Ref. [41]. A dense monolayer of particles is prepared on as glass slide. (Note that for these measurements the particles are not dispersed in water.) Next, the magnetic multilayer is deposited on top of the particle monolayer. This technique creates a hemispherical magnetic coating on each particle. In a sample prepared like this, all magnetic caps are pointing in the same direction, perpendicular to the plane of the particle monolayer.

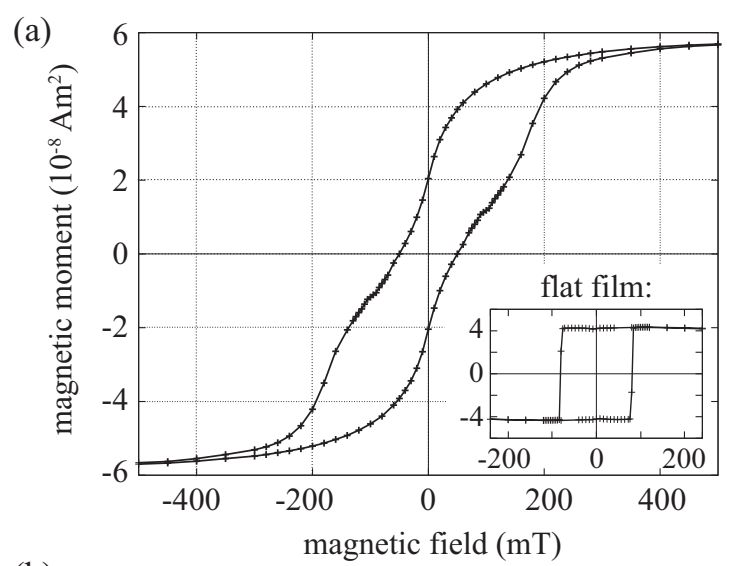

(b)

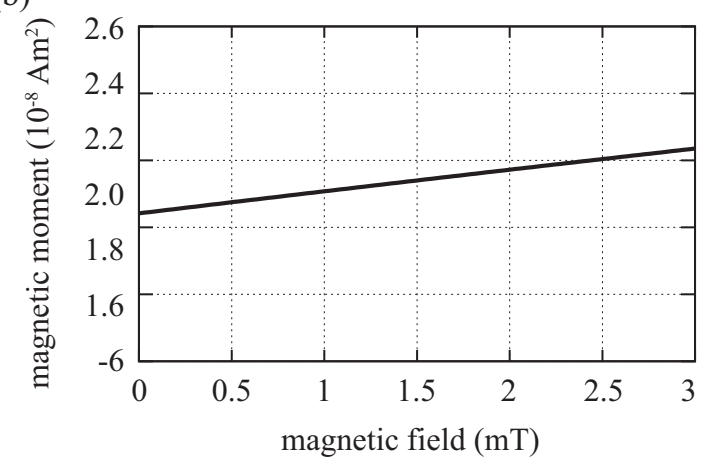

FIG. 8. Magnetization curves of a $[\mathrm{Co} / \mathrm{Pd}]_{8} / \mathrm{Pd}$ multilayer obtained by SQUID magnetometry. (a) Magnetization curve for the multilayer on an assembled particle monolayer and on a flat substrate (inset). (b) Magnified cutout of the magnetization curve of magnetic caps on the particles.

After film deposition, using a superconducting quantum interference device (SQUID), we have measured the overall magnetic moment of the monolayer of the capped silica particles as a function of applied field intensity [Fig. 8(a)]. We investigate the case where the external field points perpendicular to the particle monolayer, that is, parallel to the rotational symmetry axis of the caps. This symmetry axis also equals the direction of the net dipole moment after magnetic saturation of the particles. This is the experimentally relevant case since after particles are dispersed in water, they always align in the external field. The deposited magnetic caps with perpendicular magnetic anisotropy clearly show hysteretic behavior [Fig. 8(a)]. The magnetization exhibits a continuous switching behavior. This is different for a flat film, which abruptly switches its magnetization at its characteristic switching field [inset in Fig. 8(a)]. The difference results from the rotationally symmetric magnetic anisotropy distribution of the thin film on a spherical substrate [41]. Therefore, the magnetic state of such a capped particle is a continuous function of the applied field. However, in the particle configurations and under the low intensity of the applied fields discussed in our work, this effect can be neglected as explained next.

In the work presented here, the dispersed colloidal particles form percolated clusters with noncollinear magnetic order only below an applied field of $0.65 \mathrm{mT}$. In this range, the change of the magnetization of the cap is below $2 \%$ [Fig. 8(b)] 


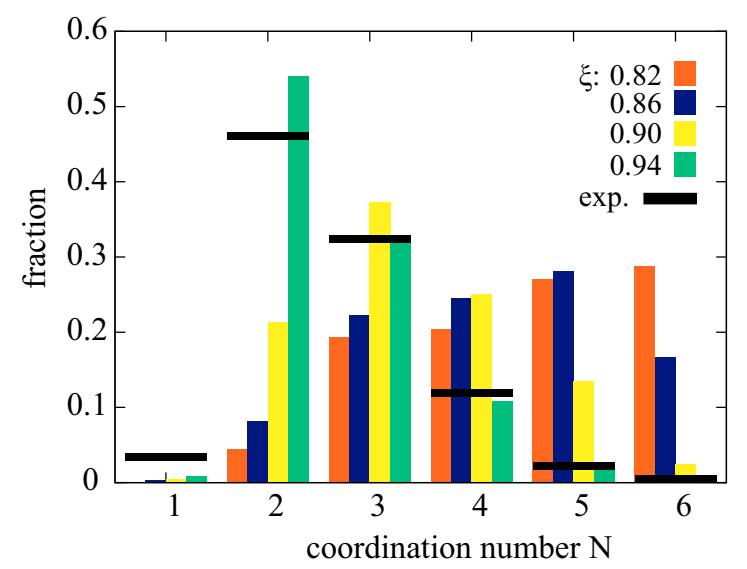

FIG. 9. Histogram of particle coordination numbers for various shifts (see legend) with $\mu=0.9$. The experimental histogram of colloidal particle coordination numbers, obtained by picture analysis, is indicated by horizontal bars.

and, thus, can be neglected in the discussion. If higher fields (up to $3 \mathrm{mT}$ ) are applied, the particles exhibit collinear magnetic order. Therefore, the associated increase of the magnetic moment is equal for all particles, and the anisotropy distribution remains rotationally symmetric. Therefore, the change in magnetic moment caused by the external fields has no qualitative impact on the magnetostatic interaction. Additionally, the particles exert magnetic stray fields on each other. The field intensity depends on the relative orientation of the caps. An upper limit for the influence of neighboring caps in a cluster can be estimated from dipole interaction. In closest possible contact, the particles are always separated by a small distance of several ten nanometers due to surface charges. The strongest magnetic flux is present at the center of the cap. Assuming a dipolar stray field with the magnetic moment $6.4 \times 10^{9} \mu_{B}$ of the caps, they exert stray fields on each other that are maximally in the range of $10 \mathrm{mT}$. Such field intensities can only affect the edge of the caps, which are affected first by external fields [66]. These regions have the lowest magnetic flux and, therefore, have only a minor contribution to the total stray field. According to Fig. 8(a), more relevant, central regions of the caps can only be switched at fields larger than $100 \mathrm{mT}$. These results suggest that a fixed anisotropy distribution in the capped particles can be assumed for the qualitative description of the assembly behavior presented in this work.

\section{Quantitative comparison between experimental and numerical field-free self-assembly}

From visual inspection of field-free self-assemblies, simulated (Fig. 3) and experimental ]Fig. 2(a), left] networks agree best for $\xi$ values of 0.9 and 0.94 . Denser, close-packed clusters result from simulations with $\xi$ values of 0.82 and 0.86 . In order to quantify the transition from dense to open networks with increasing values of the dipole shift $\xi$, we calculated the histogram of coordination numbers $N$ for the examined values of $\xi$. The results are presented in Fig. 9. The maximum value of $N$ for a particle in a monolayer is six, which corresponds to a dense hexagonal packing. If the dominant structural motif of the network is an open staggered chain or a linear chain, the most probable value of $N$ is two. Particles in branching points have $N>2$, and particles that terminate a chain correspond to $N=1$.

In Fig. 9, one can see that for low dipole shifts the dominant pattern in the network is that of hexagonal packing. Increasing $\xi$ leads to a drop of the average number of neighbors. However, only for $\xi=0.94$, the majority of particles have $N=2$. This is of special importance, as the experimental histogram exhibits a clear maximum at $N=2$ as shown in Fig. 9 with black bars. Larger shifts (not shown here) overestimate the fraction of linear segments. Based on this comparison, we conclude that the dipole shift of $\xi=0.94$ provides the best fit to model the experimental system, provided that $\mu=0.9$. It is worth noting that in the presence of extra dipoles in the $5 s d$ particle, the values of the shift are slightly larger than those reported for the $3 s d$ particle model in our previous work [37].
[1] Z. Xu, L. Wang, F. Fang, Y. Fu, and Z. Yin, Curr. Nanosci. 12, 725 (2016).

[2] S. C. Glotzer and M. J. Solomon, Nat. Mater. 6, 557 (2007).

[3] W. B. Rogers, W. M. Shih, and V. N. Manoharan, Nat. Rev. Mater. 1, 16008 (2016).

[4] J. Zhang, B. A. Grzybowski, and S. Granick, Langmuir 33, 6964 (2017).

[5] M. Marechal, B. J. Kortschot, A. F. Demirors, A. Imhof, and M. Dijkstra, Nano Lett. 10, 1907 (2010).

[6] V. Meester, R. W. Verweij, C. van der Wel, and D. J. Kraft, ACS Nano 10, 4322 (2016).

[7] I. Chakraborty, V. Meester, C. van der Wel, and D. J. Kraft, Nanoscale 9, 7814 (2017).

[8] S. Minko, Adv. Funct. Mater. 26, 3759 (2016).

[9] W. Zhao, R. Cheng, J. R. Miller, and L. Mao, Adv. Funct. Mater. 26, 3916 (2016).

[10] A. Tokarev, J. Yatvin, O. Trotsenko, J. Locklin, and S. Minko, Adv. Funct. Mater. 26, 3761 (2016).
[11] R. M. Erb, J. J. Martin, R. Soheilian, C. Pan, and J. R. Barber, Adv. Funct. Mater. 26, 3859 (2016).

[12] K. A. Arpin, A. Mihi, H. T. Johnson, A. J. Baca, J. A. Rogers, J. A. Lewis, and P. V. Braun, Adv. Mater. 22, 1084 (2010).

[13] L. Zhang, T. Petit, Y. Lu, B. E. Kratochvil, K. E. Peyer, R. Pei, J. Lou, and B. J. Nelson, ACS Nano 4, 6228 (2010).

[14] J. Wu, X. Wei, J. Gan, L. Huang, T. Shen, J. Lou, B. Liu, J. X. J. Zhang, and K. Qian, Adv. Funct. Mater. 26, 4016 (2016).

[15] J. Huang, Y. Li, A. Orza, Q. Lu, P. Guo, L. Wang, L. Yang, and H. Mao, Adv. Funct. Mater. 26, 3818 (2016).

[16] Y. Zhu, K. Kekalo, C. NDong, Y.-Y. Huang, F. Shubitidze, K. E. Griswold, I. Baker, and J. X. J. Zhang, Adv. Funct. Mater. 26, 3953 (2016).

[17] A. Chiu-Lam and C. Rinaldi, Adv. Funct. Mater. 26, 3933 (2016).

[18] F. Guzmán-Lastra, A. Kaiser, and H. Löwen, Nat. Commun. 7, 13519 (2016). 
[19] R. Messina, L. A. Khalil, and I. Stanković, Phys. Rev. E 89, 011202(R) (2014).

[20] J. Faraudo, J. S. Andreu, C. Calero, and J. Camacho, Adv. Funct. Mater. 26, 3837 (2016).

[21] S. K. Smoukov, S. Gangwal, M. Marquez, and O. D. Velev, Soft Matter 5, 1285 (2009).

[22] B. Ren, A. Ruditskiy, J. H. K. Song, and I. Kretzschmar, Langmuir 28, 1149 (2012).

[23] M. Wang, L. He, and Y. Yin, Mater. Today 16, 110 (2013).

[24] R. Messina and I. Stanković, Eur. Phys. Lett. 110, 46003 (2015).

[25] L. Rovigatti, S. Kantorovich, A. O. Ivanov, J. M. Tavares, and F. Sciortino, J. Chem. Phys. 139, 134901 (2013).

[26] S. S. Kantorovich, A. O. Ivanov, L. Rovigatti, J. M. Tavares, and F. Sciortino, Phys. Chem. Chem. Phys. 17, 16601 (2015).

[27] A. Goyal, C. K. Hall, and O. D. Velev, Phys. Rev. E 77, 031401 (2008).

[28] H. Schmidle, C. K. Hall, O. D. Velev, and S. H. L. Klapp, Soft Matter 8, 1521 (2012).

[29] G. M. Whitesides and B. Grzybowski, Science 295, 2418 (2002).

[30] J. Zhang, E. Luijten, and S. Granick, Annu. Rev. Phys. Chem. 66, 581 (2015).

[31] D. Morphew, J. Shaw, C. Avins, and D. Chakrabarti, ACS Nano 12, 2355 (2018).

[32] J. Yan, S. C. Bae, and S. Granick, Soft Matter 11, 147 (2015).

[33] B. Ren and I. Kretzschmar, Langmuir 29, 14779 (2013).

[34] G. Vega-Bellido, R. A. DeLaCruz-Araujo, I. Kretzschmar, and U. M. Córdova-Figueroa, Soft Matter 15, 4078 (2019).

[35] A. B. Yener and S. H. L. Klapp, Soft Matter 12, 2066 (2016).

[36] J. E. Martin, R. A. Anderson, and R. L. Williamson, J. Chem. Phys. 118, 1557 (2003).

[37] G. Steinbach, D. Nissen, M. Albrecht, E. V. Novak, P. A. Sánchez, S. Kantorovich, S. Gemming, and A. Erbe, Soft Matter 12, 2737 (2016).

[38] S. Sacanna, L. Rossi, and D. J. Pine, J. Am. Chem. Soc. 134, 6112 (2012).

[39] M. Klinkigt, R. Weeber, S. Kantorovich, and C. Holm, Soft Matter 9, 3535 (2013).

[40] G. Steinbach, S. Gemming, and A. Erbe, Eur. Phys. J. E 39, 69 (2016).

[41] M. Albrecht, G. Hu, I. L. Guhr, T. C. Ulbrich, J. Boneberg, P. Leiderer, and G. Schatz, Nat. Mater. 4, 203 (2005).

[42] H. J. Limbach, A. Arnold, B. A. Mann, and C. Holm, Comput. Phys. Commun. 174, 704 (2006).

[43] A. Arnold, O. Lenz, S. Kesselheim, R. Weeber, F. Fahrenberger, D. Roehm, P. Košovan, and C. Holm, in Meshfree Methods for Partial Differential Equations VI, edited by M. Griebel and M. A. Schweitzer, Lecture Notes in Computational Science and Engineering, Vol. 89 (Springer, Berlin, 2013), pp. 1-23.
[44] D. Frenkel and B. Smit, Understanding Molecular Simulation (Academic, New York, 2002).

[45] J. D. Weeks, D. Chandler, and H. C. Andersen, J. Chem. Phys. 54, 5237 (1971).

[46] S. Kantorovich, R. Weeber, J. J. Cerdà, and C. Holm, Soft Matter 7, 5217 (2011).

[47] A. I. Abrikosov, S. Sacanna, A. P. Philipse, and P. Linse, Soft Matter 9, 8904 (2013).

[48] J. J. Cerdà, V. Ballenegger, O. Lenz, and C. Holm, J. Chem. Phys. 129, 234104 (2008).

[49] A. Bródka, Chem. Phys. Lett. 400, 62 (2004).

[50] L. Rovigatti, J. Russo, and F. Sciortino, Phys. Rev. Lett. 107, 237801 (2011).

[51] H. Schmidle, S. Jäger, C. K. Hall, O. D. Velev, and S. H. L. Klapp, Soft Matter 9, 2518 (2013).

[52] S. Gangwal, A. Pawar, I. Kretzschmar, and O. D. Velev, Soft Matter 6, 1413 (2010).

[53] A. Ruditskiy, B. Ren, and I. Kretzschmar, Soft Matter 9, 9174 (2013).

[54] See Supplemental Material at http://link.aps.org/supplemental/ 10.1103/PhysRevE.100.012608 for movies on structural transformations first under in-plane fields (I $\rightarrow$ II), and then under combined in-plane and out-of-plane fields (II $\rightarrow$ III $\rightarrow$ IV); on reversible contraction and expansion of a staggered chain under saturating in-plane fields (I $\leftrightarrow$ II); and on structural transformations under out-of-plane fields $(\mathrm{I} \rightarrow \mathrm{III} \rightarrow \mathrm{IV})$.

[55] D. Heinrich, A. R. Goñi, A. Smessaert, S. H. L. Klapp, L. M. C. Cerioni, T. M. Osán, D. J. Pusiol, and C. Thomsen, Phys. Rev. Lett. 106, 208301 (2011).

[56] A. Darras, J. Fiscina, M. Pakpour, N. Vandewalle, and G. Lumay, Eur. Phys. J. E 39, 47 (2016).

[57] M. Klokkenburg, B. H. Erné, J. D. Meeldijk, A. Wiedenmann, A. V. Petukhov, R. P. A. Dullens, and A. P. Philipse, Phys. Rev. Lett. 97, 185702 (2006).

[58] P. Tierno, Phys. Chem. Chem. Phys. 16, 23515 (2014).

[59] C.-H. Chen, A. R. Abate, D. Lee, E. M. Terentjev, and D. A. Weitz, Adv. Mater. 21, 3201 (2009).

[60] K. P. Yuet, D. K. Hwang, R. Haghgooie, and P. S. Doyle, Langmuir 26, 4281 (2010).

[61] T. Nisisako, Curr. Opin. Colloid Interface Sci. 25, 1 (2016).

[62] R. M. Erb, N. J. Jenness, R. L. Clark, and B. B. Yellen, Adv. Mater. 21, 4825 (2009).

[63] I. Sinn, P. Kinnunen, S. N. Pei, R. Clarke, B. H. McNaughton, and R. Kopelman, Appl. Phys. Lett. 98, 024101 (2011).

[64] K. Sandomirski, S. Martin, G. Maret, H. Stark, and T. Gisler, J. Phys.: Condens. Matter 16, S4137 (2004).

[65] A. Fernández-Nieves, G. Cristobal, V. Garcés-Chávez, G. C. Spalding, K. Dholakia, and D. A. Weitz, Adv. Mater. 17, 680 (2005).

[66] T. C. Ulbrich, D. Makarov, G. Hu, I. L. Guhr, D. Suess, T. Schrefl, and M. Albrecht, Phys. Rev. Lett. 96, 077202 (2006). 